\title{
Research on Three-Dimensional Scramjet Inlet
}

\author{
Lian-Jie Yue*, Li-Hong Chen†, Ya-Bin Xiao, Peng Gong, Xin-Yu Changł \\ (LHD, Institute of Mechanics, Chinese Academy of Sciences Beijing, 100080 China) \\ yuelj@imech.ac.cn
}

\begin{abstract}
The three-dimensional compression scramjet inlet has been investigated by using surface oil dot visualization and numerical simulation. The research has revealed the details of the internal flow pattern, which included the structure of the shock waves, the spillage, the spatial vortical structures, and the boundary layer separations etc.. These features determined the performance of the inlet, which gave the mass flow capture ratio of 0.86 , total pressure recovery of 0.41 . The results showed that the arrangement of the shocks is critical for such kind of inlet. More researches have been carried out to investigate the effect of the cowl shape, and the results showed the flow field would be changed little for the cowls with different shapes but the same internal contraction ratios.
\end{abstract}

Key Words: three-dimensional scramjet inlet, flow pattern, surface oil dot visualization

\section{INTRODUCTION}

Three-dimensional sidewall compression scramjet inlet and two-dimensional inlet have been studied for many years. Two-dimensional inlet utilizes the top plate as the primary compression surface, offering the advantage of high mass capture at the design operating condition. While it has the difficulty in obtaining good capture characteristics over the Mach number range and dealing with the vehicle forebody boundary laye ${ }^{[1]}$. Three-dimensional sidewall compression inlet wherein flow compression is accomplished in the horizontal direction by wedge-shaped sidewalls can obtain a fairly good performance over a wide Mach number range, but its mass capture is lower at the design point ${ }^{[2,3]}$.

In order to overcome the shortage of above each kind of inlet, three-dimensional compression inlet was designed, which combined the characteristics of above two types of inlets. In theory, it can raise the mass flow capture and shorten the length of compression, compared with sidewall compression inlet. In present paper, three-dimensional compression inlet has been investigated both experimentally and computationally, focusing on its flow pattern.

\section{EXPERIMENTAL METHODS}

\subsection{Inlet Model}

The inlet included four parts: base plate, two sidewalls, and the top cowl, as shown in Fig.1. The base plate consisted of a $7^{\circ}$ ramp, followed by a further compression of $5^{\circ}$, which generated two oblique shock compressions. The compression angle of the sidewall was $7^{\circ}$ with its leading edge sweep angle of $30^{\circ}$.

Two cowls with different leading edges were designed to investigate their effect on the global performance and the flow pattern. One is rectangular, called as Cowl-I, the other wedge shape cowl, named Cowl-A, as sketched in Fig.2. Either cowl had the equivalent inlet

\footnotetext{
* Associate Professor

$\uparrow$ Associate Professor, AIAA member

$\ddagger$ Professor, AIAA member
} 
internal contraction ratio of 1.28 .

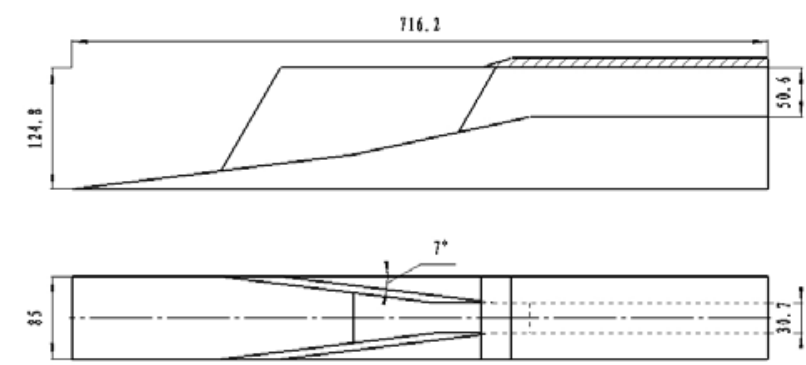

Fig. 1 Sketch of the inlet model

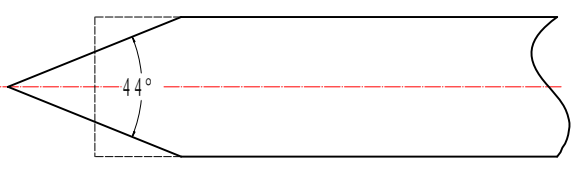

Fig. 2 Sketch of the cowl

(solid line - Cowl-A, dot line- Cowl-I)

\subsection{Experimental Facility}

The tests were conducted in the JF8A hypersonic free piston gun tunnel at Institute of Mechanics, Chinese Academy of Sciences. About 25ms of quasi-steady uniform flow condition was provided through the axisymmetric nozzle with diameter of 550mm. The nominal test conditions were free air stream with Mach number of 5.45, total pressure of $5.8 \mathrm{MPa}$, and total temperature of 660K.

\subsection{Oil Flow Visualization}

Traditional surface painted-oil approach could not be applied due to the very short test time and low-pressure condition. Then the surface oil dot visualization was adopted to trace out the surface streamlines ${ }^{[4]}$, in which Silicone oil was used as oil carrier fluid due to its low saturation vapor pressure and low viscosity. The dots of oil were pigmented by Titanium dioxide powder of about $10 \mu \mathrm{m}$ as to maximize the contrast with the black inlet surface.

\section{NUMERICAL TECHNIQUES}

Fluent 6.2 has been used as the design tool to provide a detailed characteristics and pattern of the internal flow, in which the inlet flow was assumed as fully turbulent flow. Eddy viscosity was determined using standard k- $\varepsilon$ model and wall function was employed to bridge the viscosity-affected region between the wall and the fully turbulent region. The convection terms were discretized using Second-Order Upwind Scheme.

Only half of the inlet was simulated due to its symmetrical geometry, and a portion of the external flow under the plane of the cowl was also included in order to account for the end effects in the analysis. The computation was made with a grid of 60,000 points. Grids were clustered near the leading edges and walls to better resolve the properties there, in which $\mathrm{Y}^{+}$ was nearly 30.

Pressure inlet and Pressure outlet conditions were applied at the flow entrance face and outflow plane, respectively. All solid surfaces were specified as non-slip and adiabatic wall. The initial conditions were given by assigning free stream conditions to each grid except at the wall boundaries.

\section{RESULTS AND DISCUSSIONS}

The inlet flow has been investigated by both surface oil visualization and numerical 
simulation. And the calculation was validated through the comparison between the numerical surface flow patterns with the oil flow topology. Since oil flow visualization can only figure out the flow feature near the wall surfaces, the numerical results were then used to assist the analysis the internal flow characteristics. In following figures of the oil flow pattern, $\mathrm{S}$ denotes line of separation, $\mathrm{R}$ is the line of reattachment, and $\mathrm{V}$ means vortex.

\subsection{Flow Pattern of the Inlet with Cowl-I}

Fig.3 shows the oil flow patterns of the three surfaces of the inlet with Cowl-I. The flow can be divided into three parts from upstream to downstream according to the features of the flow pattern.

The first part is the primary compression from the leading edge of the baseplate and the sidewalls, as shown in Fig.3 (a) and Fig.3 (b). The incoming flow turns upward through the baseplate induced shock, and then compressed toward the symmetric plane by the two sidewalls.

The second part is from the primary compression to the cowl, where the flow became complicated due to the shock/shock interaction and the shock/boundary layer interaction. The main characteristics in this part are the spillage and the separations. The spillage flow is revealed by the oil dots direction in Fig.3 (b). The primary separation zone (S1, R1) and a secondary separation zone (S2, R2) are caused by the interaction between the sidewall induced shock and the baseplate boundary layer ${ }^{[5,6]}$. The shock subsequently impinge on the sidewall upstream the cowl and a separation zone (S3, R3) is generated, looking like a triangle " $\nabla$ " with a larger separation near the cowl lip due to the higher back pressure. This separation would narrow the flow path, increase the inlet spillage and total pressure loss.
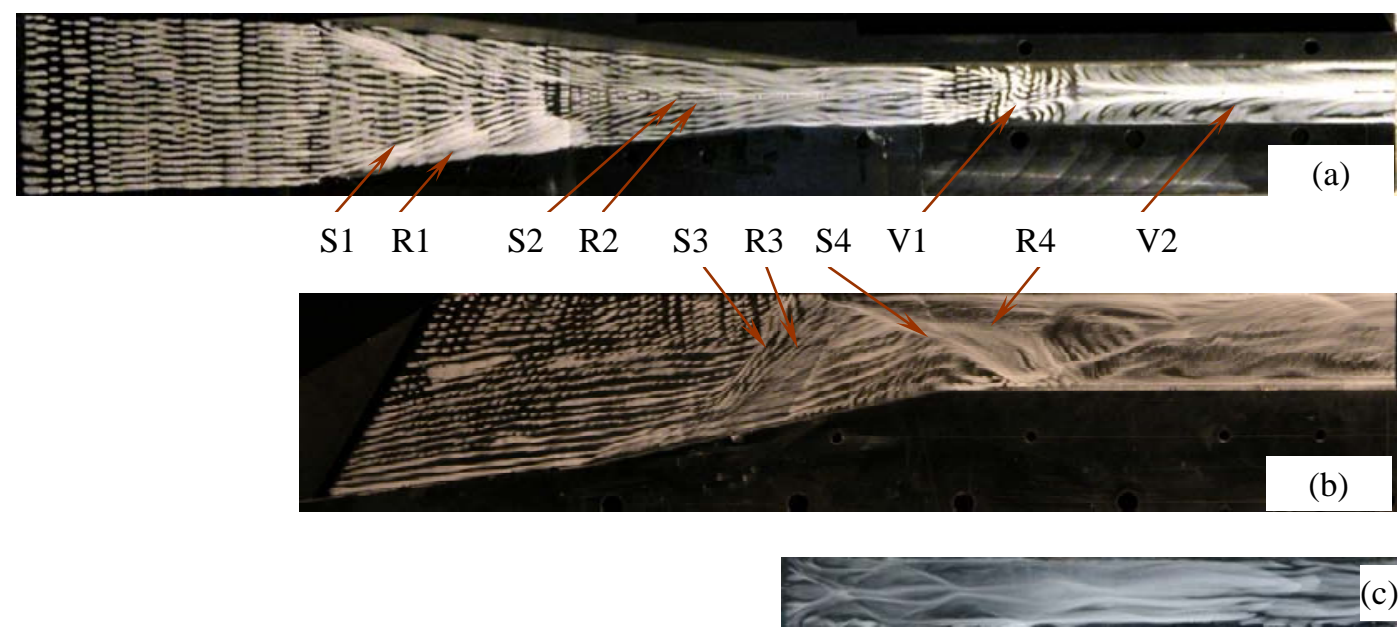

Fig.3 Oil flow patterns of the inlet with Cowl-I $\quad$ (a) baseplate (b) sidewall (c) cowl

The final part is inside the isolator, from the cowl to the exit. The cowl induced oblique shock wave also separates the sidewall boundary layer, forming line of separation S4 and line of reattachment R4, as shown in Fig.3 (b). The shock is subsequently reflected at the downstream of the expansion corner of the baseplate. Coupled with the impact of upstream expansion waves, the shock imposes high adverse pressure gradient on the baseplate boundary layer. As a result, a separation V1 is generated, as illustrated in Fig.3 (a). Apparently, 
it would make against the inlet performance, increasing total pressure loss and degrading the inlet ability of withstanding back pressure. A particular flow pattern V2 is unexpectedly observed downstream the separation V1, wherein the flow seems to rotate and sweep the oil dot toward the symmetric plane.

In order to investigate the flow pattern V2 extensively, the numerical streamlines are traced in Fig.4. It is noted that the sidewall boundary layer separated by the cowl induced shock arches downstream as a large-scale vortex off from the sidewall after impacting on the baseplate. A region with low speed and low total pressure recovery is thus yielded at bottom part of the inlet exit, as exhibited in Fig.5.

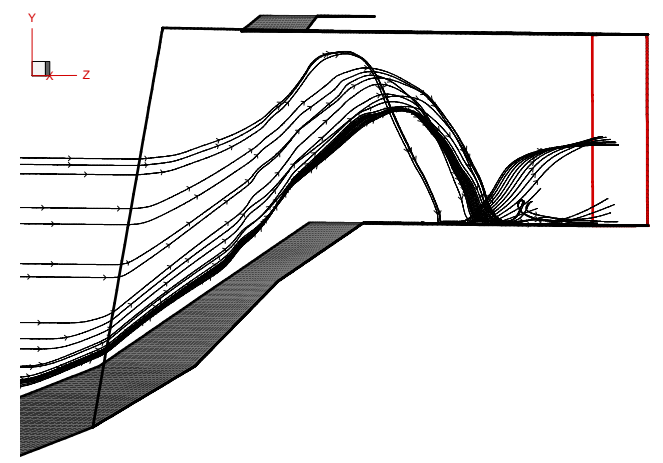

Fig.4 Sidewall boundary layer streamlines
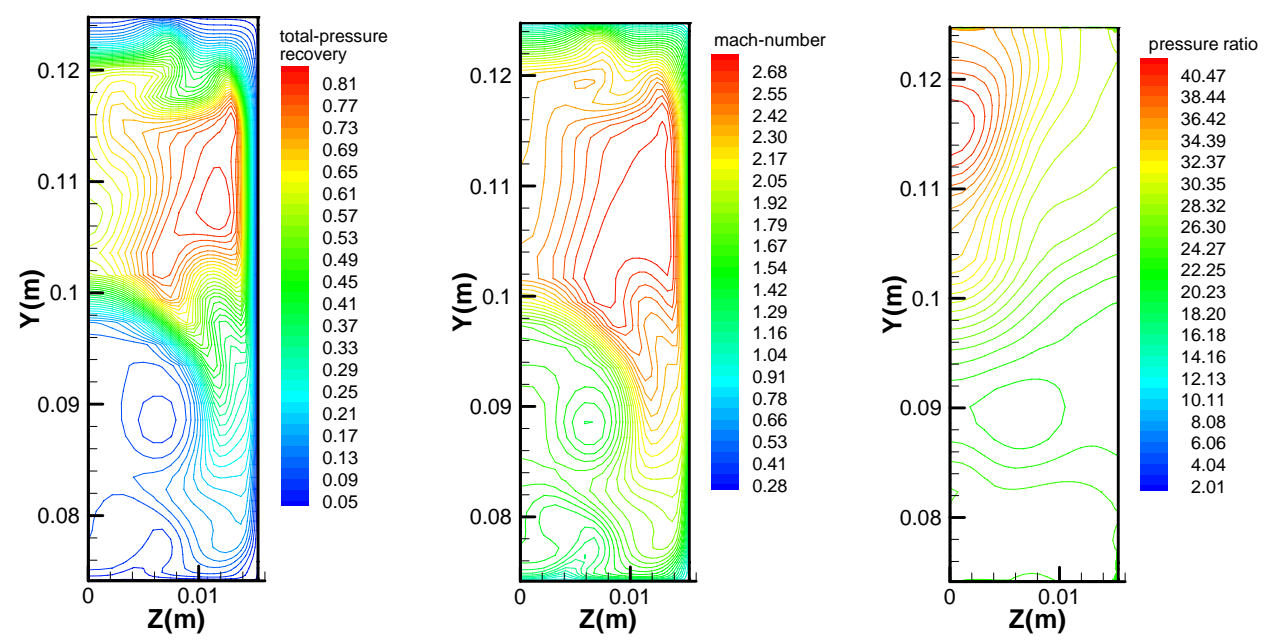

Fig. 5 contour of total pressure recovery, Mach number, static pressure ratio at the inlet exit

Fig.6 and Fig.7 further present the numerical contours of the static pressure ratio and Mach number on the symmetric plane $(\mathrm{z}=0)$, and lateral plane $(\mathrm{z}=15 \mathrm{~mm})$, respectively. A prominent characteristic exhibited in Mach-number contours on the symmetric plane, as shown in Fig.6 (b), is that a low speed layer develops from the second baseplate compression corner, while the baseplate boundary layer grows only a little on $\mathrm{z}=15 \mathrm{~mm}$ lateral plane, as shown Fig. 7(b). It is noted in the pressure contours that two sidewall induced shocks intersect with the second baseplate compression corner just near the symmetric plane. The composite impact of above shocks would contribute to growth of the baseplate boundary layer more rapidly. The other reason of the low speed layer can be attributed to the vortical motion of the baseplate boundary layer. Fig.8 exhibits the baseplate boundary layer streamlines, in which the primary separated boundary layer (S1, R1) turns toward the symmetry plane, and does not reattach but rather roll up into a clearly discernible vortical motion from the baseplate. The low speed layer then grows rapidly and extends to the inlet exit coupled with the vortical structures triggered by the cowl induced shock. 


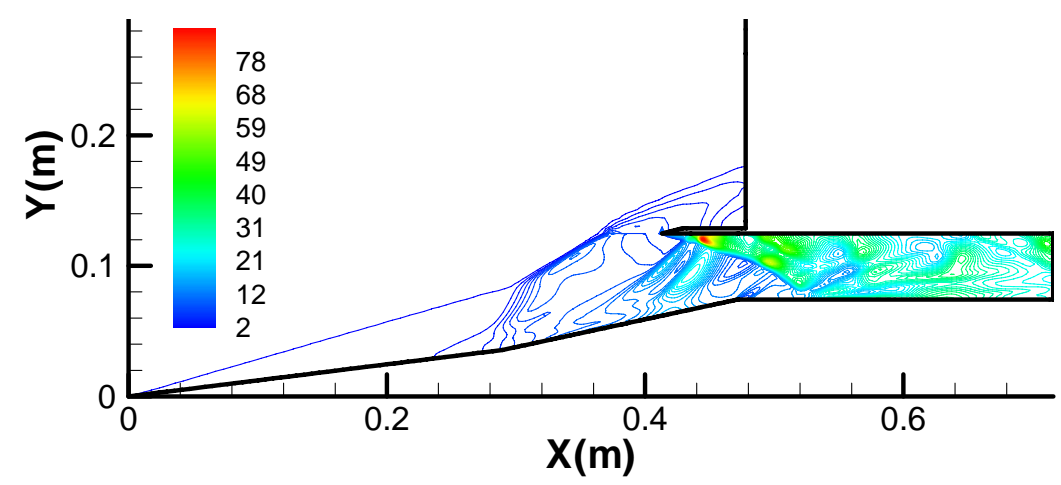

(a) Static pressure ratio

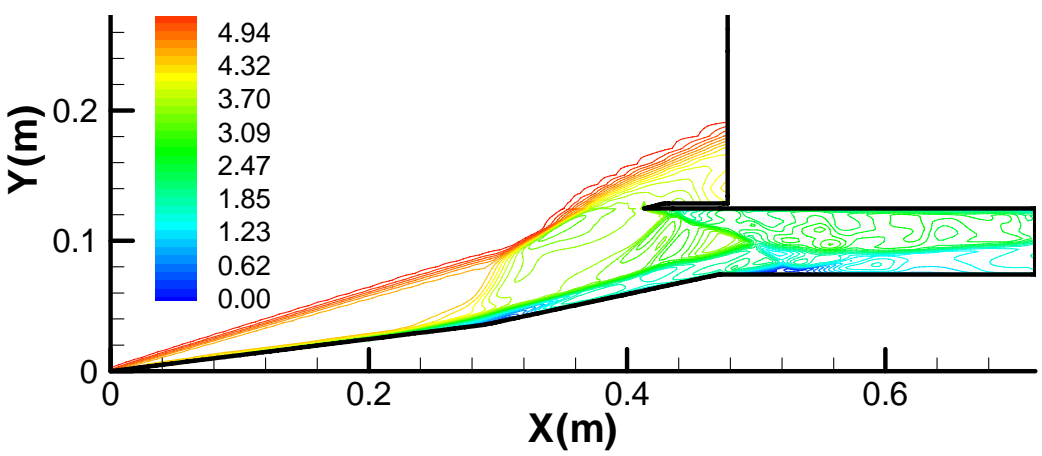

(b) Mach number

Fig.6 Contours of Mach number and static pressure on the symmetry plane ( $\mathrm{Z}=0$ )

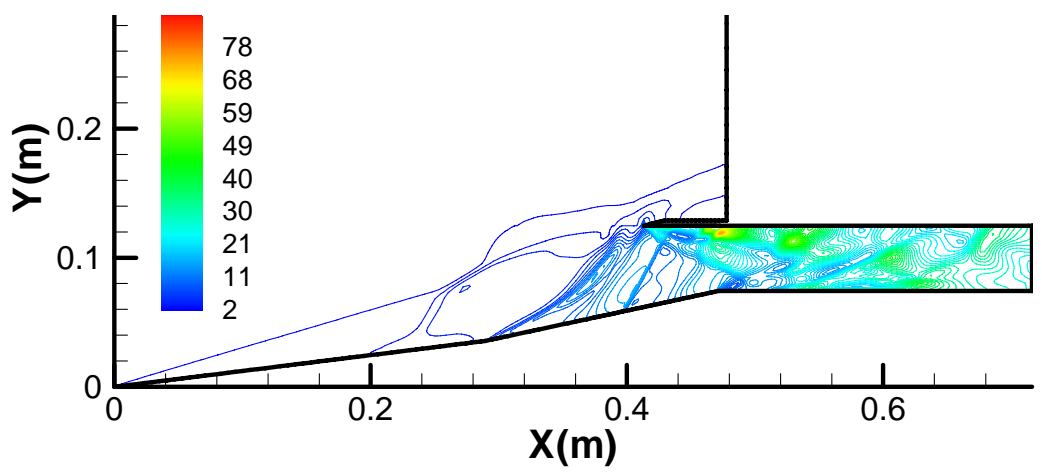

(a) Static pressure ratio

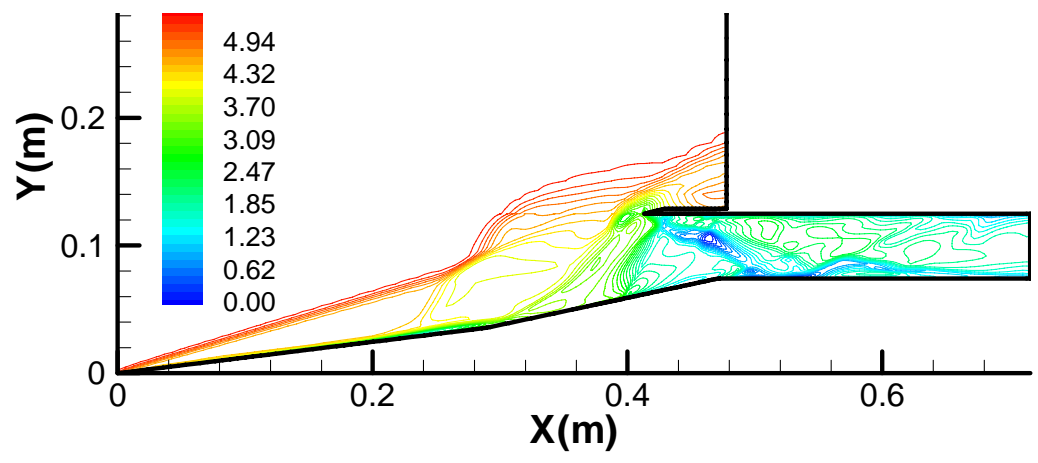

(b) Mach number

Fig.7 Contours of Mach number and static pressure on lateral plane (z=15mm)

It is observed further in Fig.7 that the shocks induced by the sidewall and second 
baseplate compression corner coalesce into a stronger shock wave near the sidewall, which just results in the formation of separation (S3, R3). By comparison, in the research on the flow pattern of sidewall compression inlet ${ }^{[7]}$, the shock emanating from $9^{\circ}$ sidewall ramp can not even separate the sidewall boundary layer at the same flow condition once the baseplate compression was absented. Therefore, it can be concluded that the design of the second baseplate compression corner is critical for removing the sidewall separation.

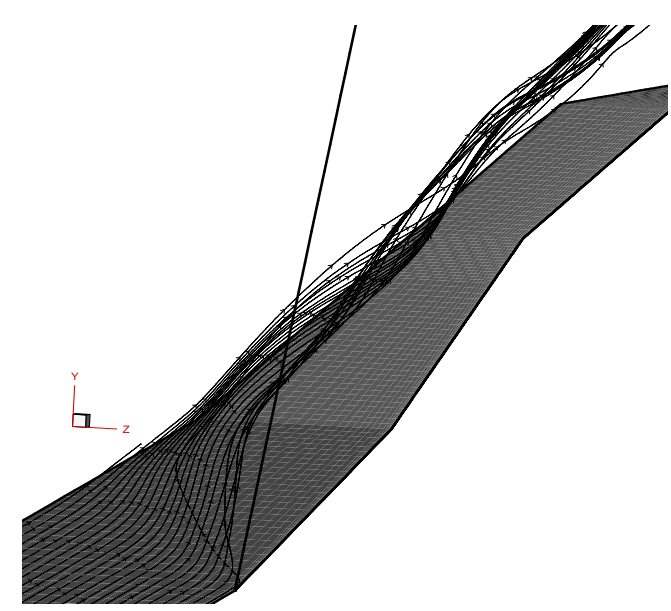

Fig.8 Baseplate boundary layer streamlines

\subsection{Analysis of the Inlet Performance with Cowl-I}

Numerical results show that the global performance of the three-dimensional compression inlet is well with the average parameters at the inlet exit: the mass flow capture ratio of 0.86 , total pressure recovery of 0.41 , Mach number of 2.3 , static pressure ratio of 29 , and static temperature ratio of 3.45. The three-dimensional compression inlet would satisfy the requirement on the contraction ratio in either horizontal or vertical direction, and is helpful in increasing total pressure recovery. Moreover, it is anticipated that the open spillage window would offer the advantage of good inlet start performance. Nevertheless, as analyzed in previous section, the flow is swirling and not uniform at the inlet exit. The design of the combustor to match with the inlet flow characteristics would be critical to the success of a scramjet engine.

It is also found that the present inlet still needs to be optimized through the analysis on its flow pattern. Its mass capture ratio is only equivalent to the sidewall compression around the design condition. The shocks should be arranged reasonably to prevent the unnecessary separation of the boundary layer.

\subsection{Effect of Cowl-A}

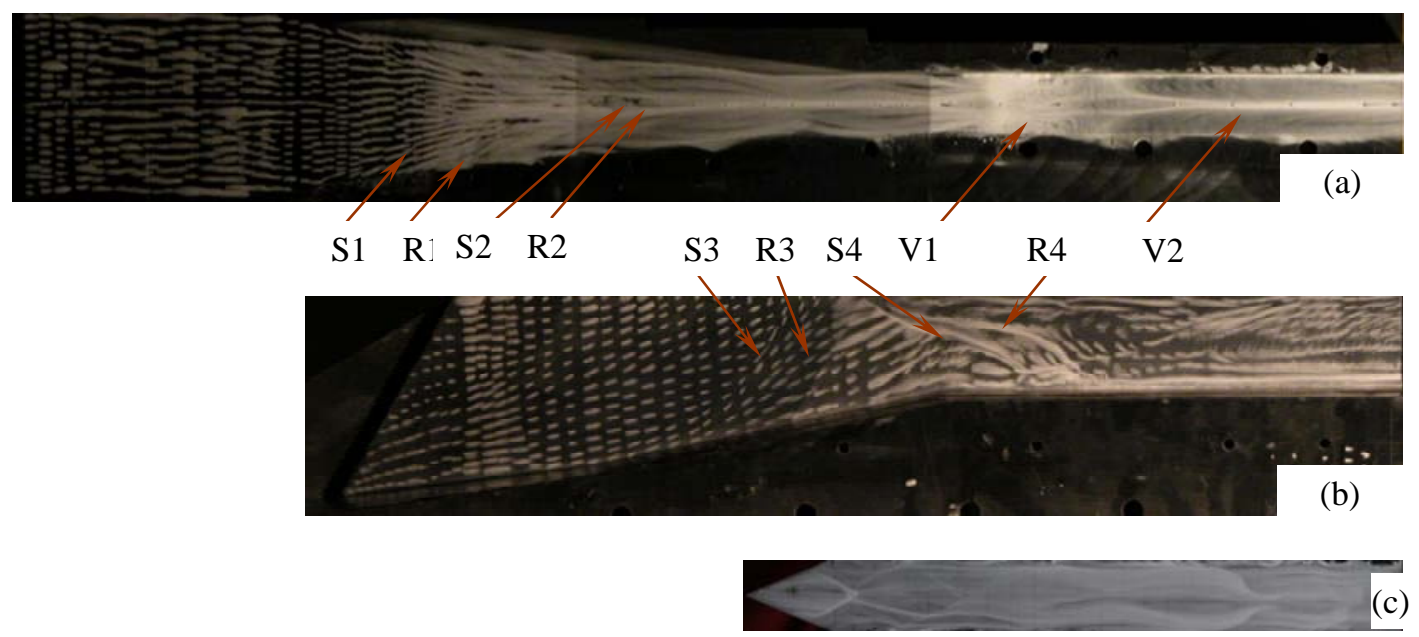

Fig.9 Oil flow pattern of the inlet with Cowl-A (a) baseplate (b) sidewall (c) cowl 
Fig.9 exhibits the surface oil flow pattern of the inlet with Cowl-A. Some prominent lines of characteristic can be noted as nearly same as in Fig.3, except the curved characteristic lines (S4, R4), which imply a 3-Dimensional curved shock. Fig.10 further demonstrates the contour of total pressure recovery, Mach number and static pressure at the inlet exit. Compared to Fig.5, the contours are very similar to that with Cowl-I, and the region with low speed and low total pressure also occurs. Numerical results also demonstrate that the global performance of inlet with Cowl-A is nearly the same as with Cowl-I. Although with different shapes, both cowls provide open flow spillage windows with the same area. Together with the nearly same flow conditions around both leading edges, the inlet flow field would be changed little.
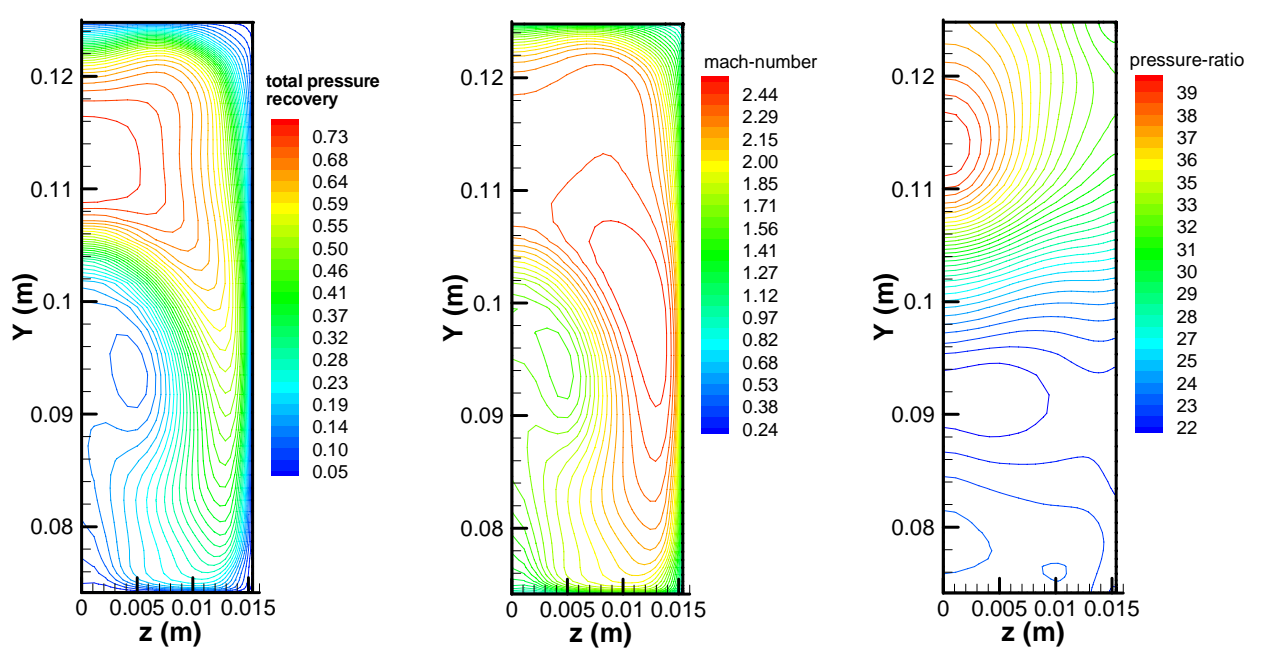

Fig.10 Contour of total pressure recovery, mach number, static pressure ratio at the inlet exit

\section{CONCLUSIONS}

The three-dimensional compression scramjet inlet has been studied by using surface oil dot visualization and numerical simulation, focusing on its internal flow pattern. Moreover, two cowls with different leading edges, one rectangular cowl, the other wedge shape cowl, has been investigated. Some conclusions can be drawn as follows:

1) Three-dimensional compression inlet is well with mass flow capture ratio of 0.86 , total pressure recovery of 0.41 . While some modification should be further made to improve its performance as several boundary layer separations and various vortical structures are observed. Especially, the arrangement of the shocks is critical for such kind of inlet.

2) Coupled with the impact of upstream expansion waves, the cowl induced shock separates the baseplate boundary layer. Moreover, the sidewall boundary layer separated by the cowl induced shock does not reattach but rather roll up into a clearly swirling flow from the wall after impacting on the baseplate, resulting in a large region with low total pressure and low Mach number at the exit. Then the design of the combustor to match with the inlet flow characteristics would be critical to the success of a scramjet engine. 
3) Near the symmetric plane, a low speed layer grows rapidly from the second baseplate compression turn to the inlet exit due to the shocks/turbulent boundary layer interaction.

4) The coalescence of shocks induced by the sidewall and second baseplate compression corner separates sidewall boundary layer upstream the cowl lip, which would seriously degrade the mass capture.

5) Although with different shapes, the cowls would little affect the flow field due to their equivalent internal contraction ratio.

\section{ACKNOWLEDGE}

The project is funded by National Natural Science Foundation of China (90305022, 10525212). The authors wish to express their appreciation to Mr. D. Qian for his help with model design and manufacture, to Mr. Z. Luo, Q. Li, C. Yu and D. Sun for their help with the experiments.

\section{REFERENCES}

[1] Carl A. Trexler, Sue W. Souders, "Design and Performance at a Local Mach Number of 6 of an Inlet for an Integrated Scramjet Concept”, NASA TN D-7944

[2] Scott D. Holland, "Internal Aerodynamics of a Generic Three-Dimensional Scramjet Inlet at Mach 10 ”, NASA TP-3476

[3] Ajay Kumar, "Numerical Simulation of Flow through a Two-Strut Scramjet Inlet", Journal of Propulsion and Power, Vol.5, No.3, May-June, 1989, pp341-345

[4] Wang Shi-Fen, Wang Yu, Liu Peng, "Surface Feature in Hypersonic Swept Shock and Boundary Layer Interaction”, ACTA Aeronautica et Astronautica Sinica, 1993, 14(9), pp449-454

[5] Doyle D. Knight, C. C. Horstman, etc., "Structure of Supersonic Turbulent Flow Past a Sharp Fin”, AIAA Journal, Vol.25, No.10, Oct., 1987, pp1331-1337

[6] J. D. Schmisseur and Datta V. Gaitonde, "Numerical Investigation of New Topologies in Strong Crossing Shock-Wave/Turbulent Boundary Layer Interactions”, AIAA 2000-0931, 38th Aerospace Sciences Meeting \& Exhibit, 10-13, Jan., 2000, Reno, NV

[7] Lian-jie Yue, An-yu Xiang, Ya-bin Xiao, Peng Gong, Li-hong Chen, Xin-yu Zhang, "Research on the Flow Pattern of Sidewall Compression Scramjet Inlet”, The 5th Asian-Pacific Conference on Aerospace Technology and Science, Oct. 30 Nov. 3, 2006, Guilin, China 